\title{
Low order controller design for continuous fluidized bed spray granulatiovn with internal product classification by robust control methods
}

\author{
Stefan Palis ${ }^{1, *}$, Andreas Bück ${ }^{1}$ Achim Kienle ${ }^{1,2}$ \\ 1 Otto-von-Guericke-Universität, \\ Universitätsplatz 2, D-39106 Magdeburg, Germany \\ 2 Max-Planck-Institut für Dynamik komplexer technischer Systeme \\ Sandtorstrasse 1, D-39106 Magdeburg, Germany
}

\begin{abstract}
This paper is concerned with stabilizing open loop unstable fluidized bed spray granulation with internal product classification by means of low order control methods. The process under investigation can be described by a population balance equation. In order to design a low order controller numerical approximation and model reduction methods are applied resulting in a low order design model. Stability when applying the low order control to the original plant can be guaranteed under certain conditions on the approximation and reduction methods and the robustness of the designed controller. The controller is validated by simulation of the nonlinear plant model.
\end{abstract}

Keywords: distributed parameter systems; low order control design ; stabilization; model reduction; Granulation ; population balance.

\section{INTRODUCTION}

Granulation is an important process in many industries, for example food, detergents, and pharmaceuticals. The aim often is the production of a solid, free-flowing powder from liquid raw materials, because powders are often easier to handle, to transport or to store than their liquid basis components. Additionally, it is possible to change properties of the particles in the powders.

One process that allows for the production of particles from liquid starting material is fluidized bed layering granulation. Here, a liquid suspension, containing the dissolved solid material, is sprayed on particles that are kept in a fluidized state by a gas flow. Due to fluidization of the particles by hot gas, the liquid in the suspension evaporates and the solid content forms a layer on the surface of the carrier particle, involving heat and mass transfer (liquid to solid, liquid to vapor) and particulate processes (growth of the layer).

The process can be run either in batch mode, which is heavily used for instance in pharmaceuticals for the application of protective layers on active ingredients, and also in a continuous way: Here the suspension is sprayed onto the particles, a growth of particles results, and by some classifying mechanism particles that have reached a certain size are removed from the process as product. In order to maintain the continuous operation, a continuous flow of new initial particles (nuclei) is required, because otherwise all particles will at some time reach the required product size and no particles remain in the apparatus.
Classification schemes can be separated into two groups: internal and external classification. Internal classification means that the particles are classified before they leave the apparatus; in contrast, in external classification particles of all possible sizes leave the apparatus, are then screened and the rejected portion of the outlet flow is fed back into the apparatus for further growth [1].

In Vreman et al. [2] the authors proposed a model for a continuous fluidized bed spray granulator with internal product classification, where nuclei are produced by spray drying, i.e. the drying conditions are such that the liquid in a fraction of all the droplets sprayed from the nozzle evaporates before it comes in contact with a particle in the apparatus. These nuclei then form particles in their own right. For certain ranges in the region of operating parameters, an oscillating behavior in product properties is observed. In view of given product specifications, this effect is unwanted. This gives a motivation of product control in particulate processes.

As particles in a process in general are not uniform, i.e. they possess a distribution with respect to characteristic properties, for example the size, the process behavior can only be described if the distributed character is taken into account. This can be done within the framework of population balance equations [5] which allows for the modeling of the temporal evolution of particle property distributions $n$ by considering the particles as individuals in a population (the granular product). The resulting balance equations are in general nonlinear partial integrodifferential equations, and thus from a system-theoretic point of view infinite-dimensional problems. 
General obstacles in the design and implementation of feedback control for distributed parameter systems are: (a) there is no complete theory for nonlinear infinitedimensional systems, (b) feedback controllers are often also infinite-dimensional systems, that have to be approximated as finite-dimensional systems in order to be implementable in process control systems, and (c) although there is a theory for finite-dimensional systems, the question always remains whether a controller designed on the basis of a finite-dimensional approximation is sufficient to guarantee closed-loop stability when it is applied to the infinite-dimensional process.

In this contribution a control scheme for the feedback control of a fluidized bed spray granulation process as presented in Vreman et al. [2] for the stabilization of the oscillating particle size distribution is presented. Starting with an infinite-dimensional populations balance model for the particle size distribution a finite-dimensional system is derived. In a further step this model is reduced in complexity by application of balanced truncation of the transfer function model, i.e. the linearization of the nonlinear finite-dimensional approximation. The total error in approximating and reducing the population balance model is then quantified by the concept of gap-metric. Following, a feedback controller for the reduced dynamic model is designed. Here the focus lies on controller structures that are easily available and accepted in industries, e.g. PI controllers. Finally, by using gap-metric results, it will be shown that the simple controller is able to stabilize the infinite-dimensional system.

\section{PROCESS MODELING}

In the following the main equations and relations derived by Vreman et al. [2] for a continuous fluidized bed spray granulation process with internal product classification as depicted in Fig. 1 are summarized.

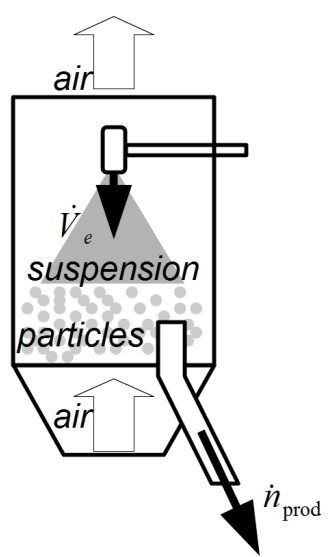

Fig. 1. Process scheme

The suspension sprayed by the nozzle leads to a growth of size of the particles in the bed. Assuming spherical particles, the characteristic size of the particles is the diameter. For the growth of particles a surface-proportional law can be derived. The main assumption in the derivation is that the droplet spreads over the total particle surface. Vreman et al. [2] modified the growth law, to account for nuclei formation by spray drying, yielding:

$$
G=\frac{2(1-b) \dot{V}_{e}}{\varrho \pi \int_{0}^{\infty} L^{2} n d L}=\frac{2(1-b) \dot{V}_{e}}{\varrho \pi \mu_{2}} .
$$

Here, $\dot{V}_{e}$ denotes the volumetric flow of suspension sprayed onto the particles, the total surface area of all particles in the bed is given by $\pi \mu_{2}$, where $\mu_{2}$ depends on the particle size distribution $n$. The fraction $b$ accounts for the formation of nuclei and depends, according to Vreman et al., on the distance of the particle bed from the nozzle With increasing bed height $h$ the free distance for the spray droplets decreases yielding a decreasing nuclei formation. The minimum for the nucleation parameter $b_{\infty}$ is reached, if the bed reaches the height of the nozzle. For larger bed heights the nucleation parameter $b$ is fixed at $b_{\infty}$. If the bed height vanishes, it is assumed that $100 \%$ of the injected suspension forms new nuclei giving a nucleation parameter $b=1$. In between these two extrema the curve is interpolated linearly.

$$
b=b_{\infty}+\max \left(0,\left(1-b_{\infty}\right) \frac{h_{n o z}-h}{h_{n o z}}\right) .
$$

The bed height can be calculated for a given bed porosity $\varepsilon$ from the granulator area and the overall particle volume.

$$
h=\frac{V}{(1-\varepsilon) A},
$$

The particles created are assumed to be normally distributed about the mean length $L_{0}$ resulting in the following birth function $B$.

$$
B=\frac{b \dot{V}_{e}}{\frac{1}{6} \varrho \pi} \frac{e^{-\frac{\left(L-L_{0}\right)^{2}}{a^{2}}}}{\int_{0}^{\infty} L^{3} e^{-\frac{\left(L-L_{0}\right)^{2}}{a^{2}}} d L}=\frac{b \dot{V}_{e}}{\frac{1}{6} \varrho \pi} n_{B}(L),
$$

The outlet flow of particles can be modeled as

$$
\dot{n}_{\text {prod }}=K T(L) n,
$$

where $K$ is the drain and $T$ the separation function due to the internal classification, which is achieved by an air sifter with a counter-current stream that separates particles with respect to their sinking velocities. Here, the following nonideal classification is assumed.

$$
T(L)=\frac{\int_{0}^{L} e^{-\frac{\left(L^{\prime}-L_{1}\right)^{2}}{a^{2}}} d L^{\prime}}{\int_{0}^{\infty} L^{3} e^{-\frac{\left(L-L_{1}\right)^{2}}{a^{2}}} d L}
$$

The population balance model for this process thus reads

$$
\frac{\partial n}{\partial t}=-G \frac{\partial n}{\partial L}-\dot{n}_{\text {prod }}+B \text {. }
$$

Starting with an initial particle size distribution as depicted in Fig. 2, which is the steady state particle size distribution for $\dot{V}_{e, 0}=16700 \frac{\mathrm{mm}^{3}}{\mathrm{~s}}$, the model shows an interesting dynamical behavior: For sufficiently high suspension injection rates and an associated bed height higher than the nozzle height, a stable steady state in the particle size distribution is reached, as shown in Fig. 3 (left). By a decrease in the suspension rate below a critical value the steady state becomes unstable and nonlinear oscillations occur as depicted in Fig. 3 (right). 
The mechanism yielding these oscillations can be described as follows:

- For a bed height smaller than the nozzle height an increased nuclei production takes place due to spray drying.

- This results in a high number of small particles and a reduced growth rate.

- After a certain time the bed height reaches the nozzle height, resulting in a small and constant production of nuclei and a higher growth rate.

- When the peak of the particle size distribution reaches the critical classifying particle radius $L_{1}$ which passes the air sifter, the associated particles are removed from the granulator. This is connected with a decrease of the bed height below the nozzle height and hence the process repeats.

In contrast, a high suspension rate results in a permanent high nuclei production, a higher growth rate and therefore a bed height being bigger than the nozzle height. Hence, after a transition a stable steady state particle size distribution is reached and no oscillations occur.

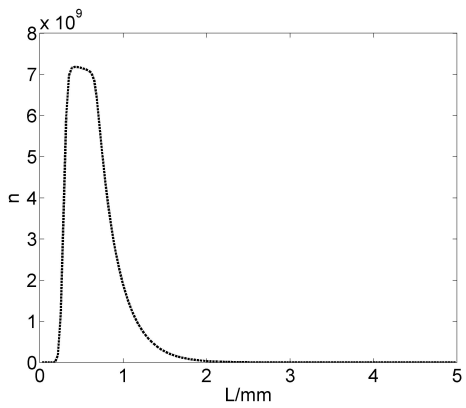

Fig. 2. Initial condition
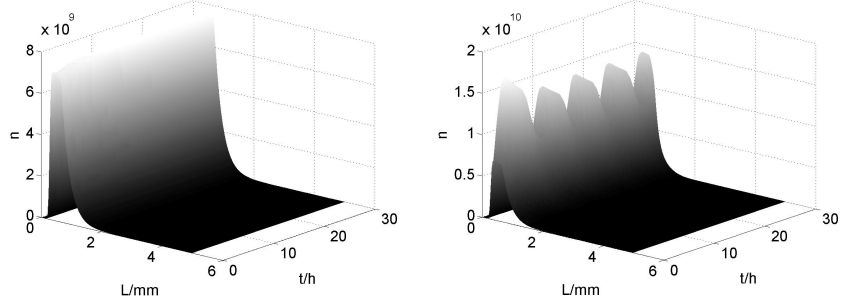

Fig. 3. Open loop Simulation in the stable (left) $\dot{V}_{e}=1.1$. $\dot{V}_{e, 0}$ and unstable (right) $\dot{V}_{e}=0.9 \cdot \dot{V}_{e, 0}$

\subsection{Control structure}

In order to stabilize the continuous fluidized bed spray granulation with internal product classification a controller will be derived using a transfer function model with the suspension rate $\dot{V}_{e}$ as the control input $u$ and the third moment of the particle size distribution $\mu_{3}$ as the controlled variable. The resulting control scheme is shown in Fig. 4.

\section{MODEL REDUCTION OF POPULATION BALANCES BY NUMERICAL APPROXIMATION}

For simulation and finite dimensional control design numerical discretization has to be applied to the presented

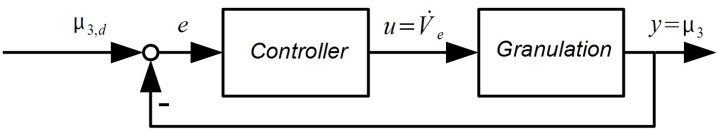

Fig. 4. Control scheme

model. Due to the fact that the presented population balance model can be written as a scalar conservation law

$$
\begin{aligned}
\frac{\partial n}{\partial t}+\frac{\partial}{\partial L}\left[G n+\int_{0}^{L}\left(\dot{n}_{\text {prod }}+B\right) d L^{\prime}\right] & =0 \\
\frac{\partial n}{\partial t}+\frac{\partial f(n)}{\partial x} & =0
\end{aligned}
$$

application of finite volume discretization is preferable. Here, convergence results are typically stated for the fulldiscrete case [3], i.e. discretization in space and time, for simulation and control design the semi-discrete case, i.e. applying the method of lines first discretizing in space and then using a possibly different method for time discretization, is more appropriate. The reasons are twofold:

(1) applying a discretization in two steps allows the use of high-order accurate time integration methods, e.g. Runge-Kutta methods, and variable time step methods.

(2) a discretization in space results in a high-order continuous-time state-space model, which can be investigated by finite dimensional continuous-time analysis and control methods, whereas a discretization in space and time results in a high-order discretetime state-space model.

In order to prove convergence of a semi-discrete approximation towards a weak solution $n$ the full-discrete case is studied first. Applying appropriate numerical discretization methods to a scalar conservation law results in a discrete conservation law in conservation form.

$$
n_{j}^{k+1}=n_{j}^{k}-\frac{\Delta t}{\Delta x}\left[F_{j}-F_{j-1}\right] .
$$

Here $F_{j}$ is the numerical flux function, which may depend on the numerical solution $n_{p}^{k}$ at time step $k$ at different grid points $p$. A special case of this general approximation scheme is the first order upwind scheme (11).

$$
n_{j}^{k+1}=n_{j}^{k}-\frac{\Delta t}{\Delta x}\left[f\left(n_{j}^{k}\right)-f\left(n_{j-1}^{k}\right)\right]
$$

In the following it will be assumed that time and space discretization $\Delta t$ and $\Delta x$ fulfill the basic CFL condition [3]

$$
\frac{\Delta t f^{\prime}}{\Delta x} \leq 1
$$

where $f^{\prime}$ is the characteristic wave propagation speed. Then the main ingredients to prove convergence of a numerical method in conservation form, here the upwind scheme, to a weak solution are total variation stability, which guarantees convergence, and consistency together with Lipschitz continuity, which gives that the limit is a 
weak solution. It can be shown that the upwind scheme gives a convergent approximation [3].

As has been stated earlier, full-discretization is not preferred from a control design point of view when focus is on continuous control design methods. Therefore the discretization is mostly done in two stages, first discretizing in space. This approach is called the method of lines. It reduces the distributed parameter system to a high dimensional system of ordinary differential equations.

$$
\frac{d u}{d t}=L(u)
$$

Here, $u$ is the spatially discretized approximation of the solution and $L(u)$ represents the discrete flux approximation of the true flux. Obviously, the semi-discrete representation (13) is equivalent to the fully-discrete method (10), when applying the forward Euler method for time discretization

$$
u^{n+1}=u^{n}+\Delta t L\left(u^{n}\right)
$$

One advantage using the method of lines approach is, that applying standard high order ODE solvers, e.g. RungeKutta methods, high temporal accuracy is possible. The open question here is, whether applying a high order ODE method still guarantees convergence, i.e. total variation stability. In the following a general Runge-Kutta scheme [4] is assumed.

$$
\begin{aligned}
u^{(0)} & =u^{n} \\
u^{(i)} & =\sum_{k=0}^{i-1}\left(\alpha_{i, k} u^{(k)}+\Delta t \beta_{i, k} L\left(u^{(k)}\right)\right) i=1, \ldots, m \\
u^{n+1} & =u^{(m)}
\end{aligned}
$$

The coefficients $\alpha_{i, k}$ and $\beta_{i, k}$ are assumed to be nonnegative, i.e. $\alpha_{i, k} \geq 0$ and $\beta_{i, k} \geq 0$. In addition by consistency the coefficients $\alpha_{i, k}$ fulfill the following equality

$$
\sum_{k=0}^{i-1} \alpha_{i, k}=1 \quad i=1, \ldots, m .
$$

This Runge-Kutta scheme is different from standard Runge-Kutta schemes typically represented by their Butcher tableau. The main advantage using this representation is that for $\alpha_{i, k} \geq 0$ and $\beta_{i, k} \geq 0$ the Runge-Kutta schemes are just a combination of Euler forward steps. The following theorem shows that if the semi-discrete scheme is TV-stable for the Euler forward step, i.e. if the equivalent full-discrete scheme is total variation stable, then the semi-discrete scheme is total variation stable applying an appropriate high order Runge-Kutta scheme.

Theorem 1. [4] If the forward Euler method is total variation stable and fulfills the CFL condition with a time step $\Delta t$, then the Runge-Kutta method with $\alpha_{i, k} \geq 0$ and $\beta_{i, k} \geq 0$ is total variation stable and fulfills the $\overline{\mathrm{CFL}}$ condition

$$
\frac{\Delta t}{\Delta x} \max _{i, k}\left(\frac{\beta_{i, k}}{\alpha_{i, k}}\right) f^{\prime} \leq 1
$$

For numerical time integration among others the following third order Runge-Kutta scheme [4] could be used

$$
\begin{aligned}
u^{(0)} & =u^{n} \\
u^{(1)} & =u^{(0)}+\Delta t L\left(u^{(0)}\right) \\
u^{(2)} & =\frac{3}{4} u^{(0)}+\frac{1}{4} u^{(1)}+\frac{1}{4} \Delta t L\left(u^{(1)}\right) \\
u^{(3)} & =\frac{1}{3} u^{(0)}+\frac{2}{3} u^{(2)}+\frac{2}{3} \Delta t L\left(u^{(2)}\right) \\
u^{n+1} & =u^{(3)} .
\end{aligned}
$$

The coefficients $\alpha_{i, k}$ and $\beta_{i}$, clearly satisfy the aforementioned assumptions.

\section{CONVERGENCE IN THE GAP METRIC}

So far, convergence of the approximate solution of the particle size distribution has been studied. However, for control design this type of convergence is not sufficient. For a successful finite dimensional control design convergence should be stated in terms of certain error bounds, which can be identified with appropriate model uncertainties. Here, we will focus on normalized coprime factor uncertainties. The motivation is that deriving a controller with an appropriate robust stability margin closed loop stability can be guaranteed embedding the linearized population balance model into a set consisting of a nominal system $G_{0}(s)$ and a set of bounded, stable uncertainties.

Therefore, in the following it is assumed that the population balance model has been linearized resulting in a linear distributed parameter system on a Hilbert space $\mathcal{X}$, where $A$ is the generator of a strongly continuous semigroup $T(t)$ and the input/output spaces are finite dimensional, i.e. $B \in \mathcal{L}\left(\mathbb{R}^{m}, \mathcal{X}\right)$ and $C \in \mathcal{L}\left(\mathcal{X}, \mathbb{R}^{n}\right)$.

$$
\begin{aligned}
& \dot{x}=A x+B u \\
& y=C x
\end{aligned}
$$

The approximations resulting from the semi-discrete upwind discretization give finite dimensional approximations.

$$
\begin{aligned}
\dot{x} & =A_{n} x+B_{n} u \\
y & =C_{n} x
\end{aligned}
$$

In the following $P_{n}$ will be the projection operator, which restricts $C$ to $C_{n}$ and gives $B_{n}=P_{n} B$. For the practical implementation the linear approximations are derived by linearization of the nonlinear semi-discretized population balance model.

From a control perspective, it would be desirable if the linear distributed parameter system could be embedded into a set consisting of a nominal system $G_{0}$ and a set of bounded, stable uncertainties. As the process shows a change of stability this should be reflected by the set of uncertainties, therefore the set of normalized coprime factor uncertainties is chosen. Here the plant is represented by its normalized left coprime factorization with additive uncertainties $\Delta M(s), \Delta N(s)$ in each factor.

$$
G(s)=\left(M(s)+\Delta_{M}(s)\right)^{-1}\left(N(s)+\Delta_{N}(s)\right)
$$

The nominal system therefore reads

$$
G_{0}(s)=M(s)^{-1} N(s) .
$$

The normalized left coprime factor uncertainty is assumed to be stable with $\left\|\left[\Delta_{N} \Delta_{M}\right]\right\|_{\infty}<\epsilon$. 
As, in contrast to multiplicative or additive model uncertainties, coprime factor uncertainties do not give a unique realization for $\Delta_{M}(s)$ and $\Delta_{N}(s)$ for a given plant to be embedded, the choice of $\Delta_{M}(s)$ and $\Delta_{N}(s)$ is an additional degree of freedom. Therefore one could derive a $\Delta_{M}(s)$ and $\Delta_{N}(s)$, which gives a minimal $H_{\infty}$-norm for $\left[\Delta_{N} \Delta_{M}\right]$.

$$
\left.\boldsymbol{\delta}_{g}\left(G_{0}, G\right):=\inf _{\left[\Delta_{N} \Delta_{M}\right] \in \mathcal{H}_{\infty}}\left\{\left\|\left[\Delta_{N} \Delta_{M}\right]\right\|_{\infty}: G\right)\right\} .
$$

As $\boldsymbol{\delta}_{g}$ is not symmetric in its arguments, the gap metric $\delta_{g}$ is introduced $[6,7]$.

$$
\delta_{g}\left(G_{0}, G\right)=\max \left\{\boldsymbol{\delta}_{g}\left(G_{0}, G\right), \boldsymbol{\delta}_{g}\left(G, G_{0}\right)\right\}
$$

Using this gap metric two systems $G_{1}(s)$ and $G_{2}(s)$ are close if the associated value of the gap metric $\boldsymbol{\delta}_{g}\left(G_{1}(s), G_{2}(s)\right)$ is close to zero, implying that both can be embedded in a family of linear models using a nominal model and a small, with respect to $H_{\infty}$-norm, coprime factor uncertainty. The maximum gap metric is one. In order to show convergence of the finite dimensional approximations $\left(A_{n}, B_{n}, C_{n}\right)$ to the linear distributed parameter system $(A, B, C)$ in the sense of the gap metric it is assumed that the sequence of approximations $\left(A_{n}, B_{n}, C_{n}\right)$ of the $\sigma$-stabilizable/detectable bounded control system $(A, B, C)$ satisfies the following assumptions.

A1 For all $x \in \mathcal{X}, \lim _{n \rightarrow \infty}\left\|P_{n} x-x\right\|=0$.

A2 For some $s \in \rho(A)$ and for all $x \in \mathcal{X}$

$$
\lim _{n \rightarrow \infty}\left\|P_{n} R(s ; A) X(s)-R\left(s ; A_{n}\right) P_{n} X(s)\right\|=0 .
$$

A3 The semigroups $T_{n}(t)$ generated by $A_{n}$ are uniformly bounded. That is, there exist $M, k$ such that

$$
\left\|T_{n}(t)\right\| \leq M e^{k t} \text { for all } n \geq N .
$$

A4 The approximations are uniformly $\sigma$-stabilizable if the original system is $\sigma$-stabilizable, i.e.

$$
\lim _{n \rightarrow \infty} K_{n} P_{n} x=K x
$$

and for sufficiently large $N$ the semigroups generated by $A_{n}-B_{n} K_{n}$ are uniformly bounded by $M e^{-\alpha t}$ for some $M>0, \alpha>\sigma$ and all $n>N$.

The following theorem then states that the sequence of finite dimensional approximations $G_{n}(s)$ of the infinite dimensional linearized population balance model $G(s)$ converge in the sense of the gap metric. Further details can be found for example in $[10,9]$.

Theorem 2. [9] Let $\left(A_{n}, B_{n}, C_{n}\right)$ be a sequence of approximations of a $\sigma$-stabilizable/detectable bounded control system $(A, B, C)$, satisfying assumptions (A1)-(A4). Then the approximating systems $G_{n}$ converge to the original system $G$ in the gap metric, i.e.

$$
\lim _{n \rightarrow \infty} \delta\left(G_{n}, G\right)=0 \text {. }
$$

\subsection{Estimation of the gap metric}

Typically the calculation of the distance between a finitedimensional approximation $G_{n}(s)$ and the original system $G(s)$ in the sense of the gap metric $\delta\left(G_{n}, G\right)$ is a very hard task. Therefore in this section an estimation procedure [8] is used. As the gap metric satisfies the triangular inequality

$$
\delta\left(G_{k}, G\right) \leq \delta\left(G_{k}, G_{k+1}\right)+\delta\left(G_{k+1}, G\right)
$$

and using the fact that the numerical approximation $G_{n}$ converges to $G$, i.e. $\lim _{n \rightarrow \infty} G_{n}=G$, yields

$$
\delta\left(G_{n}, G\right) \leq \sum_{k=n}^{\infty} \delta\left(G_{k}, G_{k+1}\right) .
$$

Assuming that the gap metric $\delta\left(G_{k}, G_{k+1}\right)$ can be bounded from above by a sequence $\bar{\delta}(k)$ for which the associated series converges, gives the following for the gap metric of the finite-dimensional approximation $G_{n}(s)$ and the original system $G(s)$ :

$$
\delta\left(G_{n}, G\right) \leq \sum_{k=n}^{\infty} \delta\left(G_{k}, G_{k+1}\right)<\sum_{k=n}^{\infty} \bar{\delta}(k) .
$$

As can be seen in Fig. 5 the sequence of gap metrics $\delta\left(G_{k}, G_{k+1}\right)$ can be bounded from above by a sequence $\bar{\delta}(k)=42 /(i+32)^{2}$ with $i=k-149$ for which the associated series converges.

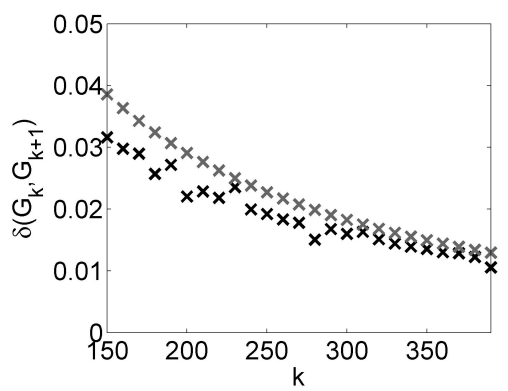

Fig. 5. Sequence of gap metrics $\delta\left(G_{k}, G_{k+1}\right)$

$$
\delta\left(G_{n}, G\right)<\sum_{i=1}^{\infty} \frac{42}{(i+32)^{2}} \leq 0.232
$$

As the interest lies on a low order controller design the finite-dimensional design model $G_{0}(s)$ should be of minimum order. Therefore, the Hankel singular values of a high order finite-dimensional approximation $G_{n}(s)$ with $n=150$ are studied. As can be seen in the diagram of the Hankel singular values $\sigma_{i}$ in Fig. 6 (left) a truncation down to order 3 is reasonable.
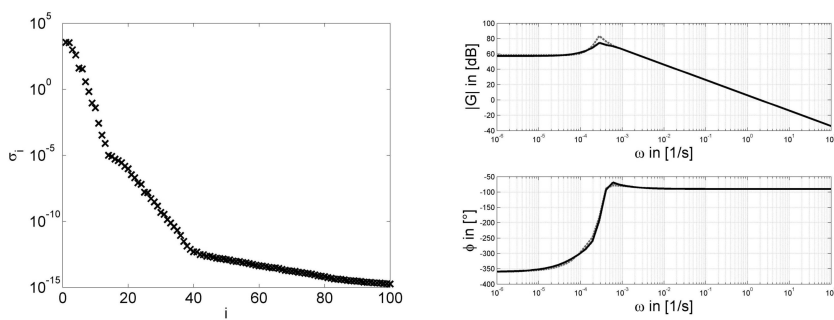

Fig. 6. Hankel singular values of $G(s)$ (left) and Bode Diagramm of $G_{n}(s)$ (solid black and $G_{0}(s)$ (dotte gray) (right)

Applying balanced truncation to the normalized coprime factors $M(s)$ and $N(s)$ of $G_{n}(s)$ and reducing each to order 3 results in a reduced system of third order.

$$
G_{0}(s) \frac{1.99\left(s^{2}+0.00028 s+3.5 \cdot 10^{-8}\right)}{(s+0.0005)\left(s^{2}-5.2 \cdot 10^{-5} s+1.2 \cdot 10^{-7}\right)}
$$

As shown in the Bode diagram in Fig. 6 (right) the additive approximation error $\left|G-G_{n}\right|$ is small over the whole 
frequency range of interest. In addition, this low order model gives a good approximation of the high order finite dimensional model in the sense of the gap metric.

$$
\delta\left(G_{0}, G_{n}\right) \leq 1 \cdot 10^{-3}
$$

\section{CONTROL DESIGN}

Using the reduced transfer function model (41) standard control design methods can be used to design a control candidate. Here, a PI controller is designed by applying standard root locus methods (Fig. 7).

$$
K(s)=8 \cdot 10^{-6} \frac{1+1.8 \cdot 10^{3} s}{s}
$$

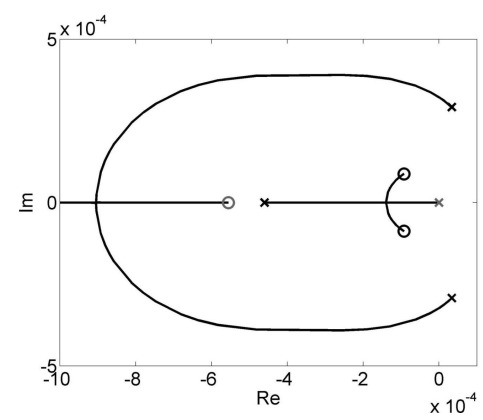

Fig. 7. Root locus of $G_{n}(s)$ (black) with PI controller (gray)

In order to assure that the designed controller stabilizes not only the reduced design model $G_{n}(s)$ but also the original distributed parameter plant $G(s)$ it has to be checked that

$$
\left\|\left[\begin{array}{c}
K \\
I
\end{array}\right](I+G K)^{-1} M^{-1}\right\|_{\infty} \leq \epsilon,
$$

where $\epsilon$ has to be smaller than the gap metric $\delta\left(G_{0}, G\right)$. An appropriate estimate can be calculated taking into account the numerical approximation error $\delta\left(G_{n}, G\right)$ and the model reduction error $\delta\left(G_{0}, G_{n}\right)$.

$$
\delta\left(G_{0}, G\right) \leq \delta\left(G_{0}, G_{n}\right)+\delta\left(G_{n}, G\right) \leq 0.233
$$

As the designed PI controller achieves a robustness margin of approximately $\epsilon=0.7$ this condition is met. Nonlinear simulations depicted in Fig. 8 show that closing the control loop after $60 \mathrm{~h}$ the suggested controller is able to stabilize the system with reasonable control effort.

\section{CONCLUSION}

A low order controller for continuous fluidized bed spray granulation with classifying product removal has been developed. For the control design the main steps are model discretization, linearization of the discretized model, model reduction, control design and verification of the controller robustness, which guarantees stability in the neighborhood of steady state. The proposed control strategy was validated by means of simulation of the nonlinear plant model. A good control performance with reasonable control effort was observed.
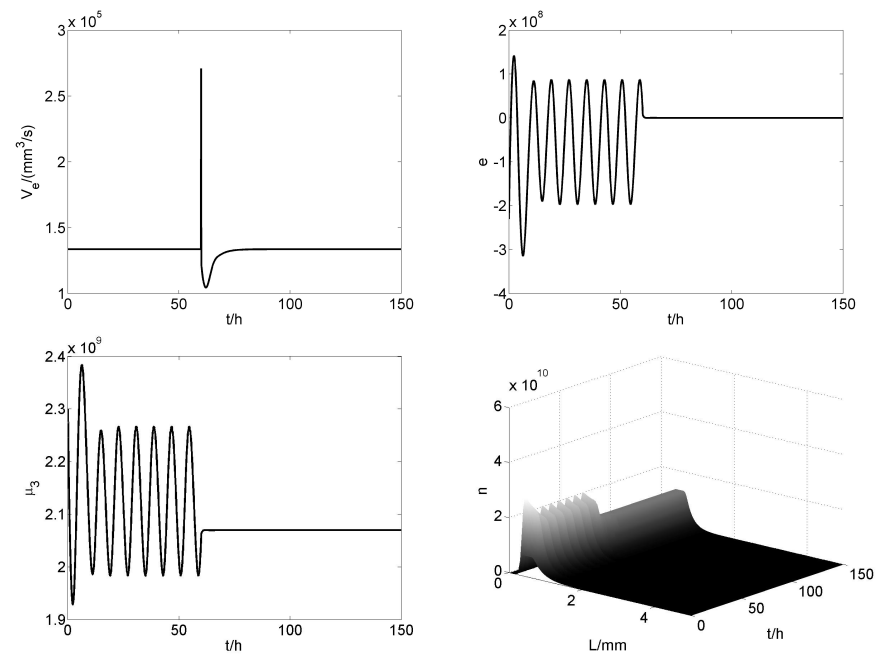

Fig. 8. Stabilization by closing the control loop at $t=60 \mathrm{~h}$

\begin{tabular}{ll}
\hline$A$ & $5 \cdot 10^{6} \mathrm{~mm}^{2}$ \\
$h_{n o z}$ & $440 \mathrm{~mm}$ \\
$\varepsilon$ & 0.5 \\
$\dot{V}_{e, 0}$ & $1.67 \cdot 10^{5} \frac{\mathrm{mm}^{3}}{\mathrm{~s}}$ \\
$b_{\infty}$ & 0.028 \\
$a$ & 0.05 \\
$L_{0}$ & $0.3 \mathrm{~mm}$ \\
$L_{1}$ & $0.7 \mathrm{~mm}$ \\
$K$ & $1.92 \cdot 10^{-4} \frac{1}{\mathrm{~s}}$ \\
\hline
\end{tabular}

Table 1. Plant parameters

\section{REFERENCES}

[1] S. Heinrich \& M. Peglow \& M. Ihlow \& M. Henneberg \& L. Mörl, Analysis of the start-up process in continuous fluidized bed spray granulation by population balance modeling, Chem. Eng. Sci. 57, 2002, pp. 43694390.

[2] A.W. Vreman \& C.E. van Lare \& M.J. Hounslow, A basic population balance model for fluid bed spray granulation, Chem. Eng. Sci. 64, 2009,pp. 4389-4398.

[3] R. J. LeVeque, Finite-volume methods for hyperbolic problems, Cambridge University Press, 2004.

[4] C.-W. Shu. A survey of strong stability-preserving high-order time discretization methods. In Collected Lectures on the Preservation of Stability under Discretization, SIAM, 2002.

[5] A. D. Randolph \& M. A. Larson, Theory of particulate processes, New York: Academic Press, Inc., 1988.

[6] G. Vinnicombe, Measuring Robustness of Feedback Systems, PhD thesis, Department of Engineering, University of Cambridge, 1993.

[7] K. Zhou \& K. Glover, Robust and Optimal Control, Prentice Hall, 1996.

[8] B. L. Jones \& E. C. Kerrigan, When is the discretization of a spatially distributed system good enough for control?, Automatica 46, 2010, pp. 1462-1468

[9] K. A. Morris, Design of finite-dimensional controllers for infinite-dimensional systems by approximation, Journal of Mathematical Systems, Estimation and Control 4, 1994, pp. 1-30.

[10] R. Curtain \& H. Zwart, An Introduction to Infinitedimensional Linear Systems Theory, Springer, 1995. 